\title{
Ethiopia: How the Care of 100 Million People Pivots on a Single Cobalt Teletherapy Machine
}

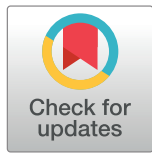

\author{
Tara J. Rick, MPAS, ${ }^{*}$ Biruk Habtamu, MD, ${ }^{\dagger}$ Aynalem Abreha, MD, ${ }^{\dagger}$ \\ Bogale Solomon, MD, ${ }^{\dagger}$ Wondemagegnhu Tigeneh, $\mathrm{MD}^{\dagger}$ \\ Mathewos Assefa, MD, ${ }^{\dagger}$ and Luca Incrocci, MD, PhD*
}

\section{*Department of Radiation Oncology, Erasmus MC, Rotterdam, Netherlands and ${ }^{\dagger}$ Department of Radiation Oncology, Addis Ababa University, Addis Ababa, Ethiopia}

Received Apr 8, 2019. Accepted for publication Jun 3, 2019.

\section{Introduction}

The Federal Democratic Republic of Ethiopia (henceforth referred to as Ethiopia) is a large country in the horn of East Africa (Fig. 1) and is composed of 9 regions, each with unique ethnic identities and linguistics. Ethiopia is the second most populated country in Africa with over 100 million inhabitants. Although it was once among the countries with the highest poverty rates in the world, in the last few decades there has been significant economic growth. ${ }^{1}$

Before the late 19th century, traditional medicine was the common practice in Ethiopia. Missionaries introduced modern medicine, and the country's earliest advancements in medicine were made under Emperor Menilek II and Haile Selassie in the 20th century. Tikur Anbessa Specialized Hospital (TASH) was opened in 1972 in Addis Ababa and was the first hospital to train doctors in the country. It is now the largest referral hospital in the country. Cancer services were initiated in 1998, 100 years after the first hospital in Ethiopia was established. TASH currently employs over 200 doctors, nearly 400 nurses, 115 other health professionals, and nearly 1000 administrative staff. It provides services to between 250,000 and 300,000 patients per year.

Corresponding author: Luca Incrocci, MD, PhD; E-mail: 1.incrocci@ erasmusmc.nl

T.J. Rick and B. Habtamu made equal contributions to this paper.

Partial funding was provided by an unrestricted research grant from Varian Medical Systems (Palo Alto, CA).
Like other low-income countries, Ethiopia has a significant burden of infectious diseases; however, along with economic advancements, communicable diseases have declined and life expectancy has increased. ${ }^{2,3}$ This has inevitably led to a shift in attention toward noncommunicable diseases as cancer has become a leading cause of mortality in adults in Ethiopia. ${ }^{4}$ The burden of cancer in women is particularly significant: Breast cancer alone accounts for a quarter of cancer incidences, followed by cervical, colorectal, nonHodgkin lymphoma, leukemia, and prostate cancers. ${ }^{5}$

\section{History of radiation oncology in Ethiopia}

The first radiation therapy (RT) treatment in Ethiopia began in 1991 and was based on kilovoltage x-rays produced by contact and orthovoltage units; this was used mainly for skin and other superficial malignancies and was administered by an internist and medical physicist at TASH (Fig. 2). Kilovoltage continued for 7 years until megavoltage RT was initiated with the launch of a dedicated oncology department at TASH, a collaboration between the Ethiopian Ministry of Health and the International Atomic Energy Agency (IAEA) (Fig. 3). The first 4 oncologists in the country included a general practitioner and 3 internal medicine physicians who were sent abroad for oncology

Disclosures: none.

Acknowledgments - We thank Mikitu Negadu, Miressa Mijena, and Elleni Yohannes for their critical input on this manuscript. 




Fig. 1. Map of Ethiopia. Source: Central Intelligence Agency website, cia.gov (public domain).

training. The first, Dr Solomon was trained in Egypt, followed by Drs Tigeneh, Abreha, and Assefa, who were all trained in South Africa. Their training consisted of a dual model that included medical and radiation oncology. These 4 doctors were the sole experts on cancer in the country for many years to follow.

As cancer care expanded, the country acquired a series of 3 cobalt machines, although none functioned simultaneously (Fig. 2). The first was basic and easy to maintain and was replaced by a more automated unit that functioned for less than 2 years before being "cannibalized" for parts to be used in the currently functioning machine. The cobalt source was changed in 2016-2 years overdue-which has improved treatment times dramatically. For the past 30 years, RT has been delivered at only 1 hospital; TASH in Addis Ababa is responsible for the radiation oncology needs of the entire county. The single-site, cobalt-only era, however, is coming to an end as 6 linear accelerators (linacs) will soon be installed in different regions of Ethiopia.

\section{The current health system in Ethiopia}

The Ethiopian health system is organized into 3 distinct structural levels: primary, secondary, and tertiary. The primary level is where a majority of health care is delivered and includes health posts, health centers, and primary hospitals; the secondary level includes general hospitals; and the tertiary level includes specialized hospitals with training programs. The referral process is a stepwise approach from primary hospital to general hospital to specialized hospital when necessary.

The financing of the health system comes from different sources including government, private pay, nongovernmental organizations, and, to a lesser degree, an insurance program. However, a majority of health care financing is provided by the governmental treasury and by individual, out-of-pocket patient expenditures for fee-for-service. The government has attempted to implement community-based health insurance that allows for care at all structural levels. Health insurance has extended to various districts and covers treatment for about a third of the population. ${ }^{6}$

\section{Oncology practice}

The country has a comprehensive national cancer plan that includes aims for prevention, early detection, diagnosis and treatment of cancer, palliative care, and cancer surveillance and research. The aim of prevention is geared toward primary prevention, which includes promoting public awareness of cancer and treatment, control of alcohol and cigarette usage, and control of infectious causes of cancer with vaccination (eg, human papillomavirus, hepatitis B virus). Early detection aims include breast self-awareness, breast examinations for all women over 18 years of age who come to a health institution for other reasons, and cervical cancer screenings every 5 years for women between 30 and 49 years old. Early diagnosis also includes

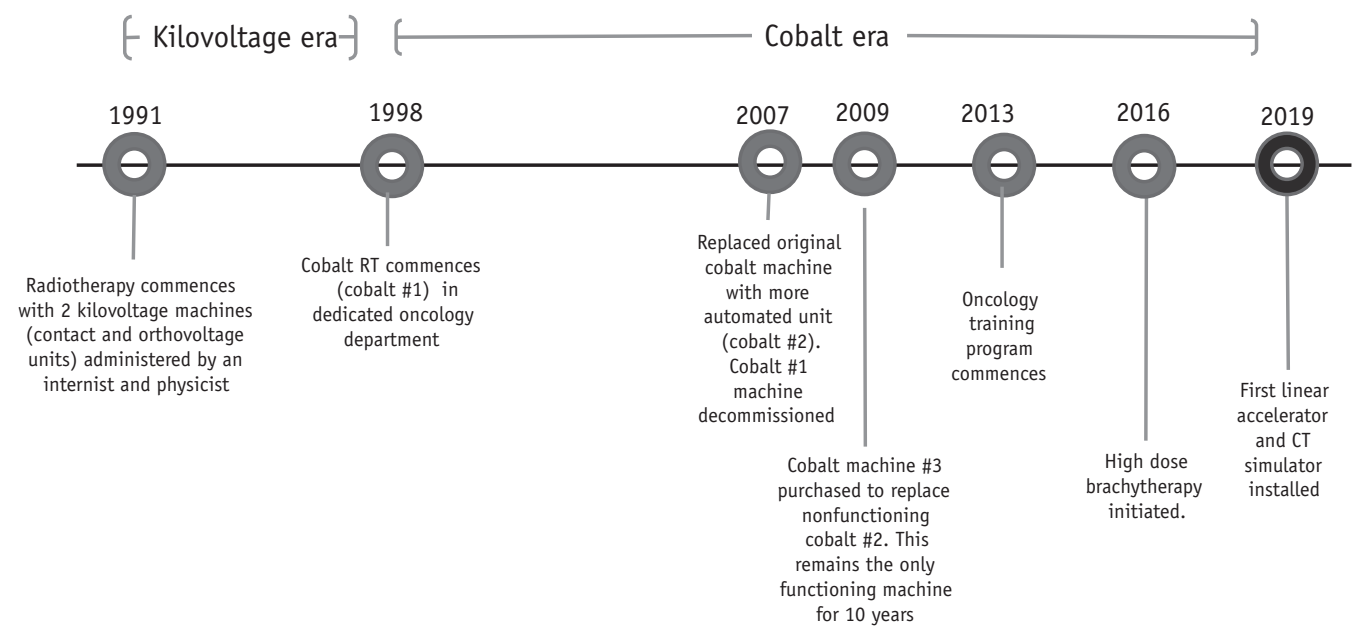

Fig. 2. Ethiopian radiation therapy: historic milestones. 


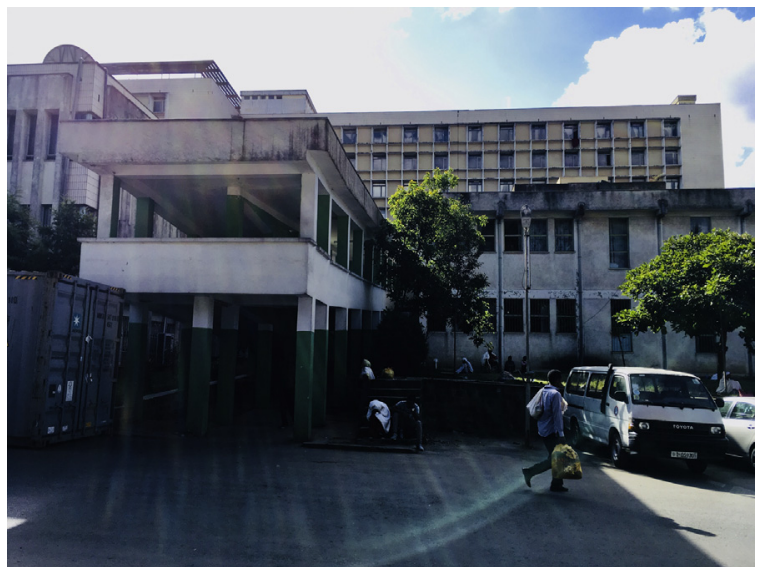

Fig. 3. Oncology department at Tikur Anbessa Specialized Hospital. Photo courtesy of author Tara Rick.

referral of patients from primary health care levels to higher levels of care in a timely manner. Diagnosis and treatment involve equipping secondary health care levels with basic technological capabilities such as cytology, x-ray, mammography, ultrasound to diagnose cancer, basic surgery and chemotherapy, and referral to the tertiary level for complex diagnostics, surgery, and RT. Palliative care goals are to have palliative care available at all health delivery systems and to develop a curriculum for palliative care disciplines (eg, doctor, nurses). The goal of cancer surveillance is achieved by establishing a national registry and local cancer registries. The final aim is to motivate research and create collaborations both regionally and internationally to improve current oncology care. ${ }^{7}$

The federal government is responsible for financing and expanding oncology services in the country. The current situation is that tertiary centers are capable of providing most standard surgical services and patients who require chemotherapy or RT services are referred to TASH, although capacity for chemotherapy at other tertiary sites is expanding. To improve the oncology care of patients at TASH, the department of clinical oncology has designated gynecologic, gastrointestinal, urologic, hepatobiliary, breast, and maxillofacial tumor boards. These tumor boards have improved communication between the departments involved in oncology care, which has made service delivery and patient care more streamlined; however, more is expected from all departments to maintain and improve these multidisciplinary meetings.

Ethiopia has a single population-based cancer registry, which was established in 2011 and is located in Addis Ababa. There are also regional hospital registries based on pathology from 5 other hospitals in the country. The registry offers great value in estimating cancer burden and identifying priorities to guide the policymaking process. In addition, the registry is an important source of information for retrospective research data.

There is a plan to establish a national organization of oncology, proposed to be called the Ethiopian Society of
Hematology and Oncology. The society is expected to receive formal acceptance from the government in 2019, and its aim will be to improve oncology care in the country. It will provide its members with a variety of academic and research opportunities to increase quality of care. This is a priority because there is currently a shortage of research owing to lack of funding and productive partnerships and inadequate research infrastructure. The majority of ongoing research is retrospective in nature, and although this research is important, clinical trials are needed to provide a basis for oncology treatments in the Ethiopian setting; currently there are no ongoing Ethiopian oncology clinical trials.

\section{Training programs}

The clinical oncology residency program was started in 2013 at TASH, and prior to this, each practicing oncologist had to be trained abroad. The residency is structured in the dual system to include both radiation and medical oncology training. This 4-year program is currently the only training program in the country and graduates 7 to 10 new oncologists per year. The training is available to those who have completed a medical doctorate degree. To receive their specialty certificate in clinical oncology at the end of the 4year curriculum, the candidate must pass clinical, oral, and written board examinations and defend a research thesis. To strengthen training in radiation oncology, the oncology department at TASH and the IAEA have developed international collaborations that have provided opportunities for residents to receive RT training on linacs abroad. Training sites currently available to residents include Egypt, Norway, and South Africa. These collaborations are important in the development of technical skills and knowledge not yet available in Ethiopia and in improving quality of care in current practice. Since the training program graduated its first class in 2017, the number of oncologists in the country has increased almost 3 -fold from the original 4 to 14 , with an additional 33 residents currently training. These residents are being trained to work in 5 new regional cancer centers that are being constructed. The initiation of the clinical oncology training program has been a vital step in increasing oncology workforce capacity in Ethiopia.

To prevent "brain drain" and loss of these new doctors to hospitals abroad, the Ethiopian government sponsors medical school tuition in exchange for an agreement of years of work, generally 2 years in a rural setting or 5 years in cities. Tertiary hospitals sponsor oncology residency for doctors they wish to employ as oncologists, and a contract may be signed agreeing they will work in that post for several more years. The retention of these new oncologists is ensuring the stability of improvements in workforce capacity.

Currently TASH also has a 2-year master's training program for oncology-certified nurses, implemented in 2016 in collaboration with the University of Oslo, Pharmacists Without Borders Norway, and IAEA. To become certified, students pass an examination and defend a research thesis. Thereafter, there is a yearly 2-week opportunity for continuing medical education for nurses. 
Table 1 Tikur Anbessa Specialized Hospital radiation oncology 2018: patients, equipment, and staffing

\begin{tabular}{|c|c|c|c|c|c|c|c|c|}
\hline Resource & Pts/y & Type of RT & Oncologists & Residents & Physicists & Dosimetrists & RTTs & OCNs \\
\hline No. & $\begin{array}{l}1700- \\
2000\end{array}$ & $\begin{array}{l}2 \text { cobalt (1 NF) } \\
1 \text { brachytherapy }\end{array}$ & 6 & $\begin{array}{c}33(6-10 \text { every y), } \\
4-y \text { program }\end{array}$ & 4 & 0 & 5 & 9 \\
\hline
\end{tabular}

Training programs for medical physicists and RT technologists are in development, also in a collaboration with the University of Oslo and IAEA. There is no dosimetry program because currently there are no dosimetrists in the country; instead, medical physicists take on this role.

\section{Radiation Therapy Care}

As mentioned, TASH is currently the only institution with RT services in Ethiopia. Its capacity incudes a single cobalt machine and a high-dose brachytherapy unit. Over 1700 patients with cancer are treated with RT every year. Although no specific data are available, Ethiopia also provides some RT services to neighboring countries such as Eritrea and Djibouti. Current staffing includes 6 oncologists, 33 residents, 4 medical physicists, 5 RT technologists, and 9 oncology-certified nurses (Table 1). Although the RT practice is broad, most patients treated with RT at TASH are women with advanced-stage gynecologic and breast cancers.

Cobalt RT is a cost-effective treatment for cancer in Ethiopia. The price of a course of RT may range from US $\$ 1.50$ to US $\$ 25$, depending on the number of fractions. For example, a single fraction of RT for bone metastasis currently costs less than US\$1.50, less than the cost of a taxi ride across Addis Ababa. On the other end of the spectrum, an entire course of RT for head and neck cancer, sarcoma, or skin cancer may cost up to US\$25. These fees are paid by patients or by insurance when applicable.

Currently, RT treatment is mainly based on 2dimensional (2D) planning with anatomic landmarks. The department has adopted 2D treatment guidelines for both curative and palliative cases. A computed tomography simulator was recently installed, and the department has started planning select cases, such as head and neck tumors, with the simulator. Because three-quarters of patients present at an advanced stage, a majority of patients are treated with palliative intent. Most treatments are two opposed anterior-posterior (APPA) fields or lateral fields. Patients undergoing curative-intent treatment are treated with standard conventional fractionation, and treatment fields are based on 2D setups. Oncologists are responsible for deciding treatment aim, fraction number, and field size. Physicists are responsible for calculation of the treatment time based on the information provided by the oncologist.

Although cobalt RT has largely been replaced by modern linacs around the world, there are some benefits to using cobalt RT in low-income countries because of its simplicity of use and maintenance and its reliability with frequent power interruptions. However, there are challenges as well, including replacing the radioactive source. The source for the cobalt machine at TASH was imported from Canada, cost over US $\$ 200,000$, and is to be replaced every 5 years. There are various issues in changing the source in a timely manner, which is why the last source was used for 2 years beyond the 5-year standard duration of use.

\section{The Challenges of Practicing Radiation Oncology in Ethiopia}

Several economic and noneconomic challenges influence cancer care in Ethiopia; some are patient related, and others are institutional. Over the past 2 decades, the number of households living below the national poverty line has decreased from nearly half to a quarter of the population. ${ }^{1,8}$ Despite this advancement, health care remains unaffordable for at least 25 million people (roughly a quarter of the population). Access to cancer care is another challenge because most Ethiopians live in rural communities and must travel long distances to seek specialized oncologic care. Even if treatment is affordable, the travel and housing costs during treatment are a burden that many patients may not be able to overcome.

Several patient and community factors also present a challenge to providing timely cancer care. These include lack of awareness of cancer (including misinformation), lack of health-seeking behaviors at the onset of symptoms, and preference to seek treatment from traditional or religious healers before moving on to conventional medicine. ${ }^{9,10}$ For example, if one believes that cancer is caused by supernatural powers, then modern medicine would not be considered the logical treatment. In addition, stigma surrounding certain cancers, such as cervical cancer, may lead to a patient and family being discriminated against or ostracized if the community believes the disease was caused by unacceptable behaviors.

Health care system barriers also provide significant challenges to cancer care in Ethiopia. First, there is currently a shortage of health care workers to accommodate the increasing cancer burden in the country (not unique to Ethiopia). This is being improved with the inauguration of the oncology training programs for doctors and nurses and the implementation of measures to prevent emigration. Second, RT infrastructure is grossly inadequate, with 1 RT machine per 100 million people. Third, at this time, chemotherapy is not included in the Ethiopian Essential 


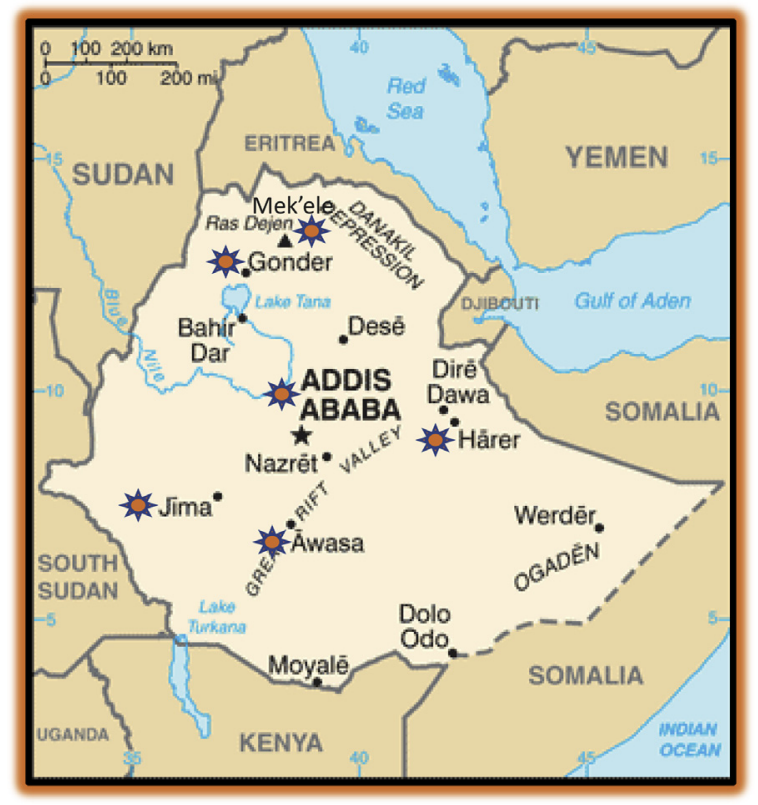

Fig. 4. Location of 6 radiation therapy facilities after 2019. Source: adapted from the Central Intelligence Agency website, cia.gov (public domain).

Medicines List, ${ }^{7}$ and although the government provides basic chemotherapy and significantly subsidizes the price, there are interruptions in availability of chemotherapy and a shortage of infrastructure capacity (chemotherapy bays). Thus, there is generally a waiting time of 2 to 3 months before patients can receive chemotherapy.

\section{The long wait for RT}

If patients requiring RT manage to get to TASH despite the aforementioned barriers, they are faced with long waiting times because service delivery depends on a single cobalt machine. The current median waiting time for all cancers, sites, and treatment intents is $\mathbf{5}$ months. Waiting times are influenced by the priority given to the large number of patients requiring palliation of debilitating symptoms such as pain or bleeding. In addition, risk reduction of urgent conditions such as pathologic fracture and cord compression in the setting of limited treatment capacity plays a major role in time allocation. This paradigm leads to a long wait for individuals with potentially curative disease, risking their conversion to the palliative category as the disease progresses. Kandelhardt et al showed that the proportion of patients with advanced-stage cervical cancer increased from $44 \%$ to $69 \%$ during the wait time. ${ }^{11}$ Nearly threequarters of patients in Ethiopia treated with RT have cancers at an advanced stage and the intent of RT is palliative. A senior oncologist applied the following quote to the current RT situation in Ethiopia: "We might not be able to add days to their lives, but we can add life to their days" (unknown author). For this reason, it is imperative to build RT capacity so waiting times will decrease and better curative-intent stratification will occur. Work needs to be done at the patient, community, and institutional levels to improve health-seeking behaviors in the general population and to streamline the process to increase early-stage diagnosis and timely access to treatment.

\section{The Future of Radiation Oncology in Ethiopia}

This year, 2019, will mark a historic milestone in Ethiopia's RT history with the addition of 5 new regional oncology facilities scheduled to open in Awasa, Gonder, Harer, Jima, and Mek'ele (Fig. 4). The government is committed to modernizing RT care and has purchased 6 new linacs (Varian Medical Systems, Palo Alto, California, USA), 1 for each new cancer center and 1 for TASH. The cobalt RT machine at TASH will continue to be used, particularly for simple palliative RT. Due to the establishment of the RT residency and oncology nurse certification training programs, it is expectated that there will be an appropriate number of doctors and nurses to staff these centers. In the future there will also be both physicist and RT technologist training programs. In addition, the University of Oslo will be sending a medical physicist and dosimetrist to provide additional training and assistance at the start of the linac program. To maintain electricity, the goal is that the linacs will have their own electric transformers and backup generators in case of electrical interruption. It is anticipated that the modern machines, which require more technical support and planning, will be more time-consuming. However, Ethiopia will be able to provide state-of-the-art RT in an increased capacity, which is expected to increase the rates of curative-intent treatment.

The era of Ethiopia's RT care pivoting around a single cobalt machine is coming to an end. Considering that the establishment of megavoltage RT treatment in Ethiopia occurred less than 2 decades ago, the tremendous progress that has been made highlights the rapid rate of development that is possible in sub-Saharan Africa. The new, more modern era is timely as cancer incidence is projected to at least double in resource-limited regions of Africa by 2040. ${ }^{12}$ We hope Ethiopia may be a successful model for other resource-limited countries as they increase RT capacity.

\section{References}

1. World Bank Group. The World Bank in Ethiopia. Available at: https://www. worldbank.org/en/country/ethiopia/overview. Accessed May 29, 2019.

2. Jembere GB, Cho Y, Jung M. Decomposition of Ethiopian life expectancy by age and cause of mortality; 1990-2015. PLOS ONE 2018; 13:e0204395

3. Misganaw A, Mariam D, Araya T. The double mortality burden among adults in Addis Ababa, Ethiopia, 2006-2009. Prev Chronic Dis 2012;9:E84.

4. Weldearegawi B, Melaku Y, Spigt M, et al. Applying the InterVA-4 model to determine causes of death in rural Ethiopia. Glob Health Action 2014;7:25550.

5. Memirie ST, Habtemariam MK, Asefa M, et al. Estimates of cancer incidence in Ethiopia in 2015 using population-based registry data. $J$ Glob Oncol 2018;4:1-11. 
6. World Health Organization. Primary health care systems (PRIMASYS): Case study from Ethiopia, abridged version. Available at: https://www.who.int/alliance-hpsr/projects/alliancehpsr_ethiopiaabridged primasys.pdf. Accessed May 29, 2019.

7. Ethiopian Federal Ministry of Health. National Cancer Control Plan 20162020. Available at: https://www.iccp-portal.org/sites/default/files/plans/ NCCP\%20Ethiopia\%20Final\%20261015.pdf . Accessed February 16, 2019.

8. World Bank Group. Ethiopia Poverty Assessment 2014. Available at: https://openknowledge.worldbank.org/handle/10986/21323. Accessed May 29, 2019.
9. Birhanu Z, Abdissa A, Belachew T, et al. Health seeking behavior for cervical cancer in Ethiopia: A qualitative study. Ethiop J Health Sci 2012;11:83.

10. De Ver Dye T, Bogale S, Hobden C, et al. A mixed-method assessment of belief and practice around breast cancer in Ethiopia: Implications for public health programming and cancer control. Glob Public Health 2011;6:719-731.

11. Kantelhardt EJ, Moelle U, Begoihn M, et al. Cervical cancer in Ethiopia: Survival of 1,059 patients who received oncologic therapy. Oncologist 2014;19:727-734.

12. Ferlay J, Ervik M, Lam F, et al. Global Cancer Observatory: Cancer Today. Available at: https://gco.iarc.fr/today. Accessed February 24, 2019. 